\title{
AVANT-PROPOS
}

\section{JEAN DERCOURT}

Secrétaire perpétuel de l'Académie des sciences

L'utilisation de l'espace exige des infrastructures très lourdes: la production des lanceurs et des satellites, des stations de lancement et de communication. De nombreux secteurs stratégiques en ont besoin : défense, télécommunications, navigation, surveillance terrestre, recherche spatiale. Certains états dont la France ont reconnu très tôt ce rôle stratégique. Plus récemment on a vu émergé dans ce secteur des états en développement rapide comme l'Inde et la Chine et la Commission européenne a commencé a y jouer un rôle qui ne pourra qu'être grandissant.

La recherche spatiale a joué un rôle clé dans le développement des techniques spatiales. Elle occupe une place très particulière au sein de la recherche française : elle résulte de la volonté de l'État, elle associe les domaines civils et militaires, elle est fortement structurée autour d'une agence de programmation et de moyens (le Centre national d'études spatiales) et d'une agence de recherche (le CNRS avec ses deux Instituts : I'Institut National des Sciences de I'Univers et I'Institut National de Physique Nucléaire et de Physique de Particules) associant des chercheurs de cet établissement et ceux des universités. Très créatrice d'innovations technologiques, elle est fortement associée au milieu industriel. Elle est intégrée aux structures analogues des autres pays européens, en une Agence spatiale européenne et elle collabore avec les pays historiquement leaders du domaine : les États-Unis, l'URSS puis, désormais, la Russie; elle s'associe aujourd'hui aux puissances nouvelles dans ce domaine : Japon, Inde, Chine et Brésil.

La recherche spatiale est une source dominante des données dans plusieurs secteurs en particulier dans les sciences de l'environnement. Dans ce secteur, à la suite des satellites dédiés à la recherche arrivent des satellites opérationnels dont les satellites de météorologie ont été le premier exemple. La recherche spatiale contribue à la définition mais est aussi utilisatrice de ces satellites. L'imagerie de plus en plus fine de toute la Terre (avec la filière française des satellites SPOT) est un autre exemple d'utilisation conjointe de l'espace pour des besoins opérationnels et scientifiques. 
La recherche spatiale a un très fort impact sur les opinions publiques qui voit grâce à elle l'état de la planète en temps réel et ouvre une nouvelle frontière à la tendance naturelle de l'humanité vers l'exploration.

Science lourde, très onéreuse, nécessitant des programmations pluriannuelles, la recherche spatiale occupe, dans la recherche française, une place originale; un ministère entier lui fut consacré. Elle ne peut être comparée qu'au domaine de la recherche nucléaire, avec ses applications civiles et militaires.

Aujourd'hui, un profond remodelage structurel de la programmation et du financement est en cours. En 2004, la Commission européenne a élaboré un Livre blanc, auquel le Comité de recherche spatiale de l'Académie des sciences a contribué par un avis.

La première partie de ce rapport expose la stratégie et la structuration de la programmation au sein de l'Europe, telles que les comprend l'Académie. La deuxième présente les forces et les faiblesses de la recherche spatiale française, analysées par disciplines, et propose des recommandations pour une bonne adaptation aux stratégies européenne et mondiale en cours d'élaboration, aux côtés des programmes nationaux de son propre ressort. Les documents analytiques élaborés peuvent être consultés sur le site de l'Académie (www.academiesciences.fr). Compte tenu de son objectif, le rapport n'a pas ou peu examiné des pans importants du domaine spatial qu'on peut regrouper sous la rubrique « la recherche pour l'espace » et qui recouvre les apports d'autre secteurs de la recherche aux technologies spatiales, pas plus que les liens entre recherche civile et militaire.

\section{Première partie}

La recherche spatiale mondiale remporte aujourd'hui de très beaux succès, conséquences des travaux et des investissements importants qui, tous, ont été à la base de découvertes scientifiques et techniques largement disséminées hors du champ spatial strict. Néanmoins, en Europe, cette recherche traverse une crise profonde :

- le vieillissement des structures et des acteurs pionniers de la recherche spatiale et le problème de leur remplacement;

- la compétition accrue avec les États-Unis où les moyens mis à la disposition de ce secteur augmentent face à une décroissance en Europe où le climat diplomatique et financier est peu favorable à une coopération équilibrée ; 
- I'affaiblissement du partenaire russe, avec lequel des programmes bilatéraux ambitieux avaient pu être développés ;

- une faible volonté de concevoir des programmes européens, liés à la volonté pour chaque État, d'exiger le « juste retour » programme par programme qui affaiblit l'Agence Spatiale Européenne ;

- une surestimation des besoins commerciaux en lanceurs de satellites de télécommunications, et la conception erronée de satellites opérationnels financés principalement par le secteur privé qui a conduit à une politique de nouveaux lanceurs ne couvrant qu'une partie des besoins en particulier pour la recherche.

Des recommandations sont proposées pour établir une politique européenne fondée, d'une part, sur un accès autonome à l'Espace et, d'autre part, sur un rapprochement avec les pays accédant depuis peu à ce domaine (Japon, Inde, Chine, Brésil). Les recommandations de cette première partie précisent, pour la recherche française, celles du Livre blanc de la Commission européenne.

\section{Seconde partie}

La seconde partie du rapport s'attache à éclaircir ce que seront les principales questions des vingt prochaines années. Un exercice de prospective est toujours délicat et rarement réussi, à une échéance de vingt ans. Mais il est, néanmoins, crédible pour les recherches en sciences spatiales, car les délais techniques, organisationnels et financiers conduisent à des opérations longues à préparer et à interpréter : une dizaine d'années est un délai minimum. La prospective à 20 ans est donc raisonnable et significative.

Certes, les aléas de l'histoire bouleversent les prévisions. La disparition de I'URSS et son évolution vers des États indépendants a réduit considérablement l'effort spatial russe, avec lequel les équipes françaises étaient liées. Les retards successifs mis à construire la station spatiale internationale se sont accumulés. Conçue en 1984, son premier module fut mis en place en 1997. L'accident de la navette Columbia, le 1 er février 2003, aboutit à l'arrêt des vols habités américains vers la plate-forme, bouleversant la programmation des champs disciplinaires utilisateurs (physique des matériaux, biologie, physiologie et médecine). L'Union européenne ne peut pas, ni ne doit, reprendre les coûts de fonctionnement induits par le désengagement américain.

Le rapport évalue, tout d'abord, pour chaque discipline, les forces et faiblesses de la recherche française. On y verra que, si le nombre des professionnels de la recherche (chercheurs des établissements, enseignants-chercheurs, 
ingénieurs et techniciens) peut être clairement défini là où l'outil spatial est le principal outil de recherche (sciences de l'environnement et du climat terrestre, astronomie, Soleil et systèmes solaire et planétaires), dans d'autres disciplines essentiellement non spatiales, l'espace joue un rôle spécifique (médecine, biologie, physique des matériaux) ou grandissant (physique fondamentale). Dans ces derniers, la communauté scientifique est plus difficile à quantifier. Après cette analyse des forces et faiblesses, une stratégie est présentée.

La société exige de mieux connaître l'état et la dynamique de la planète, et de mieux prévoir son évolution. Les États, ou groupements d'États (dont l'Union européenne) fondent sur ce questionnement des pans entiers de leur politique scientifique et technologique. La détermination, la part anthropique de la situation actuelle, de son dynamisme et de son évolution vraisemblable, doit être clairement identifiée au sein des processus observés, résultant de la géodynamique de la planète depuis des millions d'années, dès avant l'aube de l'humanité.

Cette exigence implique que, comme pour la navigation et la géodésie et pour la météorologie, de nouveaux engins spatiaux opérationnels soient conçus et financés à l'échelle européenne par des agences dédiées à partir des résultats des travaux scientifiques ayant permis un développement technologique.

La connaissance de l'origine de la vie et la recherche des planètes " habitables ", font écho à des préoccupations fondamentales de l'humanité. C'est aussi par ces programmes que les opinions publiques des pays concernés s'attachent légitimement et avec force à la recherche spatiale.

Les investissements impliqués par cette recherche sont considérables. Les autorités devront avoir à cœur l'équilibre entre le financement des moyens envoyés dans l'espace et ceux mis en place pour le traitement, l'archivage à long terme et la distribution des données. Il apparaît en particulier que l'exploitation scientifique optimale de celles-ci reste sous dimensionnée en Europe par rapport aux efforts Nord Américains dans un contexte où les données sont, légitimement, mises rapidement à la libre disposition de la communauté scientifique mondiale.

De nouveaux champs s'ouvrent à la recherche spatiale pour résoudre des questions qui lui sont propres. L'espace est un des champs ou l'analyse de systèmes complexes et les développements en mathématiques, informatique et automatique sont essentiels. Il importe d'en tenir compte dans les l'organisation de la recherche pour l'espace entre les organismes. 